\title{
Análise de percepção de atores de Ciência, Tecnologia e Inovação em sistemas regionais e ambientes de inovação
}

\author{
Maria Angélica Jung Marques Doutoranda em Engenharia e Gestão do Conhecimento. Fundação Parque Tecnológico Itaipu, Brasil - angelica@pti.com.br \\ Vivian Costa Alves Doutoranda em Engenharia e Gestão do Conhecimento. Universidade Federal de Santa Catarina (UFSC), Brasil - vca2@uol.com.br \\ Roberto Carlos dos Santos Pacheco \\ Silvio Dagoberto Orsatto \\ Doutor. Universidade Federal de Santa Catarina (UFSC), Brasil - rpacheco@egc.ufsc.br \\ Doutorando em Engenharia e Gestão do Conhecimento. Universidade Federal de Santa Catarina (UFSC), Brasil - silvio.orsatto@uol.com.br
}

\section{RESUMO}

A análise de percepção consiste no levantamento de opinião de atores partícipes de um sistema complexo acerca de diferentes fatores que afetam sua participação e protagonismo. Recentemente a técnica foi utilizada para levantar a opinião de empresários, acadêmicos, gestores públicos e representantes da sociedade civil organizada em seis mesorregiões do Estado de Santa Catarina, no Brasil. Para tal, foi concebido o Framework ReCIS com 8 dimensões e 62 fatores de análise de percepção. Neste artigo, verifica-se a perspectiva de aplicação do mesmo Framework em outro sistema estadual de Ciência, Tecnologia e Inovação (CTI) e em outra unidade de análise, parques tecnológicos, onde também atuam atores de múltiplos matizes. $\mathrm{O}$ artigo analisa as dimensões e fatores do Framework ReCIS e verifica as necessidades de alterações para sua aplicabilidade nesses novos contextos. Como conclusão pôde-se verificar que, mediante adaptações epistêmicas e de linguagem, a aplicabilidade não somente é viável como recomendável, com benefícios aos gestores de CTI em ambientes de inovação e em sistemas regionais de CTI.

Palavras-chave: Sistema de Ciência, Tecnologia e Inovação; Desenvolvimento Regional; Sistema de Inovação; Ambientes de Inovação.

\section{Perception analysis of players in regional Science, Technology and Innovation systems and innovation habitats}

\begin{abstract}
Perception analysis is an opinion survey carried out among players of a complex system about factors that interfere in their participation and contribution. This technique was recently applied to reveal the opinion of business people, academic, public managers and civil society members in six regions of Santa Catarina state, in Brazil. The study developed a Framework called ReCIS, with 8 dimensions and 62 factors of perception analysis. In this article we study the perspective of application of this Framework in another regional system of science, technology and innovation (STI) and in another unit of analysis and technological parks, where there are also players from multiple origins. The article analyzes the Framework ReCIS dimensions and factors and verifies the need for modification for its appliance in these new domains. We have concluded that, if submitted to epistemic adaptations and content modifications, the application is not only feasible but recommended, bringing benefits to managers of STI in innovation habitats and regional systems of STI.
\end{abstract}

Keywords: Science, Technology and Innovation Systems; Regional Development; Innovation System; Innovation Habitats. 


\section{INTRODUÇÃO}

Em um sistema de Ciência Tecnologia e Inovação (CTI) participam instituições públicas e privadas: empresas, academia e governo. Quando virtuoso, esse arranjo permite que um país (ou uma região) realize avanços econômicos, sociais e tecnológicos, tanto qualitativa como quantitativamente (Alves, 2013). Para tal, gestores das diferentes áreas e instituições devem avaliar resultados das políticas, identificar demandas e novos caminhos a serem adotados em prol do fortalecimento de CTI.

No setor público, uma das práticas adotadas tem sido a realização de conferências de CTI, onde os diferentes atores do sistema são convidados a avaliar e a propor melhorias nos diferentes fatores que afetam os sistema técnicocientífico e de inovação. No Brasil, em 2010, os governos estaduais promoveram conferências estaduais que, posteriormente, tiveram seus resultados e proposições levados nas cinco regiões geográficas do Brasil, em conferências regionais relatadas na Conferência Nacional.

Em Santa Catarina, a Conferência de CTI está prevista na lei estadual de inovação n. 14.328/08. Até 2015 foram realizadas 4 conferências. Para sua quinta edição, a Fundação de Amparo à Pesquisa e Inovação do Estado de Santa Catarina (FAPESC) decidiu financiar um projeto de levantamento das percepções dos atores regionais de CTI, de modo a permitir comparabilidade entre as regiões e memória para futuras edições. O projeto da V CECTI ficou sob a responsabilidade da Rede $\operatorname{ReCIS}^{1}$, que elaborou metodologia para análise das percepções dos atores regionais de CTI e, também, para levantamento e classificação de propostas de melhorias feitas por esses atores. O chamado Framework ReCIS foi aplicado nas seis mesorregiões do $\mathrm{Estado}^{2}$, com o levantamento de percepções de grupos de trabalho compostos por empresários, acadêmicos, gestores públicos e representantes da sociedade civil organizada, que também elaboraram 450 propostas de melhoria dos sistemas regionais de CTI de Santa Catarina (Pacheco et. al, 2016).

Neste artigo, objetivamos analisar o potencial de aplicabilidade do Framework ReCIS em outro estado do País, bem como em um parque tecnológico, habitat de inovação que, a exemplo de sistemas regionais de CTI, é composto por atores de CTI de múltiplas naturezas e missões. Para tal, inicialmente apresentamos, de forma sintética, o Framework ReCIS e, posteriormente, discutimos os fatores que demandam adaptações para sua aplicabilidade nos contextos mencionados.

\section{FRAMEWORK ReCIS}

Segundo Pacheco et al. (2016), o Framework ReCIS é composto por dois modelos, um para análise de percepção dos atores sobre seu sistema regional de CTI e outro para elucidação e classificação das propostas ofertadas por esses atores para melhoria desse sistema elencadas pelos seguintes itens: dimensões, fatores e critérios de análise de percepção do Framework ReCIS.

A percepção de atores de CTI é avaliada em 8 dimensões, sendo três associadas a fatores capacitores de CTI, duas ligadas a potencializadores de CTI e as três últimas referentes aos fatores geradores de valor em CTI. Cada uma das dimensões de análise é dividida em critérios, aos quais são associados fatores, sendo cada fator avaliado por critérios associados a perguntas-referência cuja resposta se enquadra em escala Lickert de 5 pontos (Pacheco et. al., 2016). Para a aplicação do módulo de análise de percepção o método prevê a formação de equipes de atores de CTI, compostas por empresários, acadêmicos, gestores públicos e representantes da sociedade civil organizada. Em seções de discussão e consenso e divididos conforme a dimensão de análise de CTI (i.e, da Institucionalização à Inovação), os grupos respondem as questões de percepção e montam gráficos radares para apresentação e discussão em plenária. O resultado é a elaboração, apresentação e refinamento de gráficos em escalas comparáveis entre as diversas mesorregiões do Estado.

Uma vez levantadas as percepções, os grupos são convidados a elaborarem propostas de melhoria de seus sistemas regionais de CTI. Para tal, o Framework ReCIS possui um segundo módulo e uma segunda dinâmica de grupo, agora para a anotação e classificação de propostas.

\footnotetext{
${ }^{1}$ ReCIS - A Rede Catarinense de Inovação e Conhecimento Sustentáveis é liderada pelo Programa de Pós-Graduação em Engenharia e Gestão do Conhecimento (EGC), da Universidade Federal de Santa Catarina (UFSC), que configura arranjos multi-institucionais para tratar desafios estratégicos em gestão, mídia e engenharia do conhecimento. No projeto da V CECTI, a ReCIS teve a participação do Instituto Stela (que coordenou o projeto web da conferência) e das instituições que receberam os encontros regionais: UFSC e IFSC (Florianópolis), UNIVALI (Itajaí), Instituto Jourdan e SOCIESC (Jaraguá), UFFS e UNOCHAPECO (Chapecó), UNESC (Criciúma) e UDESC (Lages).

${ }^{2}$ As Mesorregiões são subdivisões dos estados que congregam diversos municípios de uma área geográfica com similaridades econômicas e sociais. Foram criadas pelo Instituto Brasileiro de Geografia e Estatística - IBGE e são utilizadas para fins estatísticos e de planejamento público, não constituindo, portanto, entidades políticas ou administrativas. 0 Estado de Santa Catarina possui seis mesorregiões.

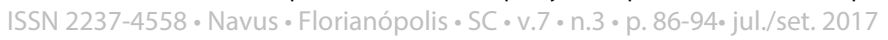


Para fins de classificação das proposições dos atores no contexto dos sistemas regionais de CTI, o Framework ReCIS utiliza os seguintes componentes de sistemas complexos: Capital Humano (propostas voltadas aos atores individuais e institucionais); Capital Relacional (propostas ligadas a relações estabelecidas pelos atores de CTI), Capital Social (propostas relacionadas aos contextos socioculturais e ao produto das relações entre atores de CTI), Capital Estrutural (propostas voltadas às estruturas físicas e intangíveis para geração de valor em CTI), Governança (propostas ligadas ao marco regulatório e ao planejamento em CTI) e Indutores de CTI (propostas de ações, instrumentos e dinâmicas para o sistema de CTI). No modelo ReCIS cada proposta tem um ou mais fatores de percepção associados (conforme Tabela 1), o que permite analisar as propostas tanto quanto à sua natureza como ao problema a que se dirige. Com isso, o Framework ReCIS dispõe de múltiplas dimensões de análise e de comparabilidade entre os diferentes sistemas regionais em que é aplicado, pois é possível comparar tanto as percepções como as sugestões ofertadas.

\section{Tabela 1: Dimensões, fatores e critérios do framework}

\begin{tabular}{|c|c|c|c|c|}
\hline Dimensões de análise & Definições & Fatores de análise & Definições & Critérios de análise \\
\hline CAPACITORES & $\begin{array}{l}\text { São dimensões } \\
\text { estruturais a um } \\
\text { sistema de CTI }\end{array}$ & 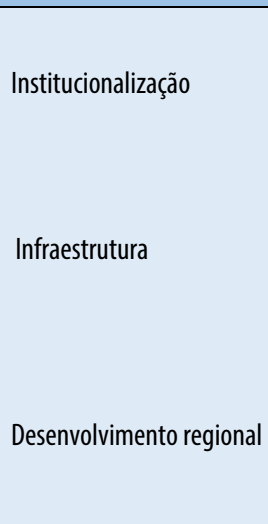 & $\begin{array}{l}\text { Identifica como uma região se encontra em } \\
\text { relação à existência e atuação das instituições } \\
\text { cuja missão se projeta sobre o sistema de CTI. }\end{array}$ & $\begin{array}{ll}\text { 1. } & \text { Diversidade institucional } \\
\text { 2. } & \text { Autonomia institucional } \\
\text { 3. } & \text { Influência dos atores } \\
\text { 4. } & \text { Relações de confiança } \\
\text { 5. } & \text { Infraestrutura básica } \\
\text { 6. } & \text { Mobilidade e transporte } \\
\text { 7. } & \text { Comunicações } \\
\text { 8. } & \text { Contexto regulatório } \\
\text { 9. } & \text { Acesso a recursos financeiros } \\
\text { 10. } & \text { Sistema de saúde } \\
\text { 11. } & \text { Inclusão social } \\
\text { 12. } & \text { Empreendedorismo } \\
\text { 13. } & \text { Cultura e entretenimento } \\
\text { 14. } & \text { Diversificação setorial }\end{array}$ \\
\hline $\begin{array}{l}\text { POTENCIALIZADORES } \\
\text { DE EFICIÊNCIA }\end{array}$ & $\begin{array}{l}\text { São dimensões de } \\
\text { empoderamento de um } \\
\text { sistema de CTI. }\end{array}$ & $\begin{array}{l}\text { Mercado } \\
\text { Educação }\end{array}$ & $\begin{array}{l}\text { Capacidade de geração econômica } \\
\text { Formação e atração de profissionais } \\
\text { especializados }\end{array}$ & $\begin{array}{l}\text { 15. Caracterização das empresas } \\
\text { 16. Relações de trabalho } \\
\text { 17. Estrutura } \\
\text { 18. Incentivos } \\
\text { 19. Efeito tributário } \\
\text { 20. Educação básica } \\
\text { 21. Oferta de educação superior e } \\
\text { 22. Recursos humanos }\end{array}$ \\
\hline $\begin{array}{c}\text { FATORES GERADORES } \\
\text { DE VALOR }\end{array}$ & $\begin{array}{l}\text { São fatores diretamente } \\
\text { relacionados à CTI da } \\
\text { região. }\end{array}$ & Ciência & $\begin{array}{l}\text { Verificam as bases instaladas e a capacidade } \\
\text { das organizações regionais de CTI aplicarem } \\
\text { novas tecnologias e criarem oportunidades } \\
\text { de geração de valor em sua região }\end{array}$ & $\begin{array}{ll}\text { 23. } & \text { Recursos humanos } \\
\text { 24. } & \text { Infraestrutura } \\
\text { 25. } & \text { Incentivos } \\
\text { 26. } & \text { Redes de colaboração } \\
\text { 27. } & \text { Pesquisa e produção intelectual } \\
\text { 28. } & \text { Geração de tecnologia } \\
\text { 29. } & \text { Tecnologia na gestão } \\
& \text { empresarial } \\
\text { 30. } & \text { Tecnologia no mercado } \\
\text { 31. } & \text { Tecnologias sociais } \\
\text { 32. } & \text { Transferência de tecnologia } \\
\text { 33. } & \text { Perfil de inovação } \\
\text { 34. } & \text { Profissionais de inovação } \\
\text { 35. } & \text { Indutores de inovação } \\
\text { 36. } & \text { Propriedade intelectual (PI) }\end{array}$ \\
\hline
\end{tabular}

Fonte: ReCIS, adaptada pelos autores, 2016.

Neste artigo nosso foco de estudo está no Módulo de Análise de Percepções (conforme Tabela 1). Nas próximas seções verificamos a aplicabilidade das dimensões, fatores e critérios em dois contextos diferentes: um outro sistema regional de CTI (de outro estado brasileiro) e em outra unidade de análise de atores de CTI (um parque tecnológico). 


\section{APLICAÇÃO DO FRAMEWORK ReCIS EM OUTROS AMBIENTES}

Considerando a importância da avaliação das políticas públicas em CTI e de seus resultados nos sistemas de CTI, seja em nível nacional, regional ou mesmo local, a pergunta em questão é se o Framework poderia ser aplicado a outros ambientes. Para fins de análise nesse artigo, consideramos a possibilidade de aplicação do módulo de análise de percepção do Framework em dois outros ambientes.

A análise de percepção pode ter vieses quali e quanti, sendo que o processo decisório, geralmente de gestores, depende, em grande parte, da percepção que os mesmos têm de si, da situação organizacional e do contexto geral em que estão inseridos (Kable, 1983). Estudos em análise de percepção são utilizados desde a década de 70 (Kable, 1979) tanto na medição dos requisitos de trabalho quanto na preferência das pessoas na tomada de decisão. Na abordagem do presente trabalho, se utiliza a análise de percepção de atores de sistemas de CTI em relação aos ambientes em que estão inseridos.

Assim, primeiro em um ambiente similar em termos de território será o Estado da Bahia, na região Nordeste do Brasil. O segundo, em um ambiente de inovação bem menor, em termos geográficos, mas concentrado em termos dos fluxos e inter-relações entre os atores, considerando o ambiente de um Parque Tecnológico.

\subsection{PERSPECTIVAS DE APLICAÇÃO DO MODELO RECIS NO ESTADO DA BAHIA}

O estado da Bahia tem aproximadamente 15 milhões de habitantes nos seus 417 municípios (IBGE, 2015) e é constituído por (07) sete mesorregiões geográficas: Extremo Oeste Baiano, Vale do São Franciscano da Bahia, CentroNorte, Nordeste, Metropolitana de Salvador, Centro-Sul e Sul Baiano.

No que se refere à composição da estrutura de Governo em CTI, o Estado conta com a Fundação de Amparo à Pesquisa do Estado da Bahia - FAPESB, (criada em 2001) e com a Secretaria de Ciência, Tecnologia e Inovação- SECTI (criada em 2003). Além disso, instituições do sistema " $\mathrm{S}$ ", banco de fomento estadual, centros de pesquisa e as universidades compõem a rede de atores de seu sistema de CTI.

No setor acadêmico, o sistema baiano de CTI é composto por 11 universidades, sendo 6 federais (Universidade Federal da Bahia - UFBA, Recôncavo Baiano - UFRB, Vale do São Francisco - UNIVALE; Oeste da Bahia - UFOB; Sul da Bahia - UFSB e Universidade da Integração Internacional da Lusofonia Afro-Brasileira - UNILAB); 4 estaduais (Universidade Estadual da Bahia - UNEB, Feira de Santana - UEFS, Sudoeste da Bahia - UESB e Santa Cruz - UESC) e 1 privada (Universidade Salvador - UNIFACS).

Como marco legal, o Estado sancionou sua Lei de Inovação no 11. 174/08, em 2008. Essa lei se constituiu como um fato de grande relevância para o Sistema Estadual (Alves, 2013). A Lei não menciona as diretrizes para elaboração da política de CTI, mas a lei de criação da SECTI, Lei no. 8.897/2003 menciona esta Secretaria como a responsável pela coordenação, direção, formulação e implementação da Política Estadual de Ciência, Tecnologia e Inovação.

A última conferência de CTI do estado foi realizada em 2010, sendo esta a sua III conferência. A metodologia adotada foi a promoção de debates em cinco eixos temáticos: Sistema nacional e estadual de ciência tecnologia e inovação; Inovação nas sociedades e nas empresas; Pesquisa, desenvolvimento e inovação em áreas estratégicas; Ciência, tecnologia e inovação para o desenvolvimento social; e Clima, meio ambiente e energia. Cada eixo temático gerou como resultado proposições para o estado, que foram apresentadas em seguida na Conferência Nacional no mesmo ano (SECTI, 2010).

O documento apresentado na Conferência Nacional gerou proposições que não chegaram a ser base para elaboração de uma nova política de CTI, como era esperado pelos participantes da conferência. Com isso, a primeira e última política (ainda vigente) foi publicada logo após a criação da SECTI, no ano de 2004.

Neste artigo, analisa-se a possibilidade de aplicação do Framework ReCIS no cenário estadual baiano. A primeira observação está na comparabilidade entre dimensões já verificadas em análises no contexto baiano e a estrutura conceitual do Framework ReCIS. Essa comparação já permite indicar que as dimensões de análise ReCIS atendem a requisitos necessários para a formulação de políticas governamentais observadas na Bahia em discussões passadas. Em sua maioria, os critérios estão adequados para serem adotados de modo a examinar a percepção dos atores participantes no sistema de CTI da Bahia. As alterações para cada dimensão de análise são:

- Institucionalização: Adicionar o critério relações interinstitucionais para contabilizar o número de atores que compõem o sistema de CTI da Bahia, sendo pertinente avaliar, além da confiança entre os atores, a intensidade das ações realizadas, particularmente quanto ao alinhamento ou sobreposições entre estes atores. 
- Mercado: Adicionar o critério postos de trabalho para identificar a percepção dos participantes em relação à oferta, especialmente para avaliar a percepção em relação a postos para trabalhos mais qualificados. Já que existe uma tendência de as pessoas mais qualificadas migrarem para regiões com postos de trabalhos compatíveis com sua formação ou capacitação.

- Educação: Substituir o componente recursos humanos por critério mais específico para o sistema baiano atual, a oferta de cursos stricto sensu, visando identificar a percepção em relação a oferta dos cursos de pósgraduação stricto sensu no Estado (dado que, atualmente, são poucos e são os programas que possuem nota igual ou superior a 5 na CAPES).

- Inovação: Adicionar o item oportunidades para inovação para identificar a percepção em relação às oportunidades que o Estado oferece para que sejam desenvolvidas inovações, seja no ambiente acadêmico ou empresarial, sendo importante obter a percepção em relação ao ambiente propício ou não para inovar.

Tabela 2: Dimensões, fatores e critérios do Framework aplicados ao Estado da Bahia

\begin{tabular}{|c|c|c|c|c|}
\hline Dimensões de análise & $\begin{array}{l}\text { Fatores de } \\
\text { análise }\end{array}$ & Critérios de análise & Critérios modificados & Justificativa \\
\hline \multirow{3}{*}{ CAPACITORES } & Institucionalização & $\begin{array}{l}\text { 1. Diversidade institucional } \\
\text { 2. Autonomia institucional } \\
\text { 3. Influência dos atores } \\
\text { 4. Relações de confiança }\end{array}$ & $\begin{array}{l}\text { 1. Diversidade de instituições } \\
\text { 2. Autonomia institucional } \\
\text { 3. Influência dos atores } \\
\text { 4. Relações de confiança } \\
\text { 5. Relações Interinstitucionais* }\end{array}$ & $\begin{array}{l}\text { Para avaliar a percepção } \\
\text { em relação a integração } \\
\text { e sobreposições de } \\
\text { ações dos atores do } \\
\text { sistema de CTI. }\end{array}$ \\
\hline & Infraestrutura & $\begin{array}{l}\text { 5. Infraestrutura básica } \\
\text { 6. Mobilidade e transporte } \\
\text { 7. } \\
\text { 8. Comunicações } \\
\text { 9. }\end{array}$ & 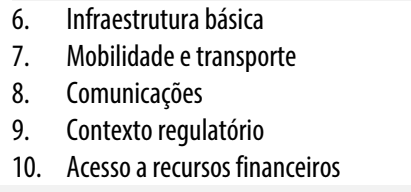 & Não há modificação. \\
\hline & $\begin{array}{l}\text { Desenvolvimento } \\
\text { regional }\end{array}$ & $\begin{array}{l}\text { 10. Sistema de saúde } \\
\text { 11. Inclusão social } \\
\text { 12. Empreendedorismo } \\
\text { 13. Cultura e entretenimento } \\
\text { 14. Diversificação setorial }\end{array}$ & $\begin{array}{l}\text { 11. Sistema de saúde } \\
\text { 12. Inclusão Social } \\
\text { 13. Empreendedorismo } \\
\text { 14. Cultura e entretenimento } \\
\text { 15. Diversificação setores econômicos* }\end{array}$ & Não há modificação \\
\hline \multirow[t]{3}{*}{$\begin{array}{c}\text { POTENCIALIZADORES } \\
\text { DE EFICIÊNCIA }\end{array}$} & Mercado & $\begin{array}{l}\text { 15. Caracterização das empresas } \\
\text { 16. Relações de trabalho } \\
\text { 17. Estrutura } \\
\text { 18. Incentivos } \\
\text { 19. Efeito tributário }\end{array}$ & $\begin{array}{l}\text { 16.Caraterização das empresas } \\
\text { 17. Relações de trabalho } \\
\text { 18. Postos de trabalho* } \\
\text { 19. Estrutura } \\
\text { 20. Incentivos diretos e indiretos } \\
\text { 21.Efeito Tributário }\end{array}$ & $\begin{array}{l}\text { Migração das pessoas } \\
\text { qualificadas para outras } \\
\text { regiões do país. }\end{array}$ \\
\hline & Educação & $\begin{array}{l}\text { 20. Educação básica } \\
\text { 21. Oferta educação superior e técnica } \\
\text { 22. Recursos humanos }\end{array}$ & $\begin{array}{l}\text { 22. Educação básica } \\
\text { 23. Oferta educação superior e técnica } \\
\text { 24. Oferta de educação strictu sensu* }\end{array}$ & $\begin{array}{l}\text { Poucos programas de } \\
\text { pós-graduação. }\end{array}$ \\
\hline & Ciência & $\begin{array}{ll}\text { 23. } & \text { Recursos humanos } \\
\text { 24. } & \text { Infraestrutura } \\
\text { 25. } & \text { Incentivos } \\
\text { 26. } & \text { Redes de colaboração } \\
\text { 27. } & \text { Pesquisa e produção intelectual }\end{array}$ & $\begin{array}{l}\text { 25. Recursos humanos especializados* } \\
\text { 26. Infraestrutura } \\
\text { 27. Incentivos } \\
\text { 28. Redes de colaboração } \\
\text { 29. Pesquisa e produção intelectual }\end{array}$ & Não há modificação \\
\hline \multirow[t]{2}{*}{$\begin{array}{c}\text { FATORES GERADORES } \\
\text { DE VALOR }\end{array}$} & Tecnologia & $\begin{array}{l}\text { 28. Geração de tecnologia } \\
\text { 29. Tecnologia gestão empresarial } \\
\text { 30. Tecnologia no mercado } \\
\text { 31. Tecnologias sociais } \\
\text { 32. Transferência de tecnologia }\end{array}$ & $\begin{array}{l}\text { 30. Geração de tecnologia } \\
\text { 31. Tecnologia na gestão empresarial } \\
\text { 32. Tecnologia no mercado } \\
\text { 33. Tecnologias sociais } \\
\text { 34. Transferência de tecnologia }\end{array}$ & Não há modificação \\
\hline & Inovação & $\begin{array}{ll}\text { 33. } & \text { Perfil de inovação } \\
\text { 34. } & \text { Profissionais de inovação } \\
\text { 35. } & \text { Indutores de inovação } \\
\text { 36. } & \text { Propriedade intelectual (PI) }\end{array}$ & $\begin{array}{l}\text { 35. Perfil de inovação } \\
\text { 36. Profissionais de inovação } \\
\text { 37. Indutores de inovação } \\
\text { 38. Propriedade intelectual (PI) } \\
\text { 39.0portunidades para Inovação* }\end{array}$ & $\begin{array}{l}\text { Avaliar a percepção em } \\
\text { relação a oferta de } \\
\text { oportunidades para } \\
\text { inovar. }\end{array}$ \\
\hline
\end{tabular}

Fonte: elaborado pelos autores, 2016.

\subsection{PERSPECTIVAS DE APLICAÇÃO DO MODELO RECIS EM UM PARQUE TECNOLÓGICO}

A segunda análise de aplicabilidade do Modelo ReCIS está na possibilidade de sua aplicação em um habitat de inovação, dado que esse é, também, um sistema complexo com múltiplos atores de CTI. Para tal, consideraremos a 
perspectiva de aplicação do Modelo ReCIS no Parque Tecnológico Itaipu - denominado PTI Brasil. Esse Parque foi criado em 2003 em Foz do Iguaçu - município do território oeste paranaense (Brasil), como um ambiente interativo integrador de diversos atores e entidades, englobando a quádrupla hélice, inserindo-se no papel fundamental da sociedade civil no processo de inovação. A instituição gestora do Parque é a Fundação Parque Tecnológico Itaipu (FPTI) - Brasil, criada em 2005.

A missão do PTI o caracteriza como ator aglutinador e indutor do processo de desenvolvimento regional, fundamentado na "ação na interseção" (Sotuyo, 2014). Para tal, uma de suas estratégias tem sido identificar os pontos de interesse comum das instituições do território, incluídas as que habitam o Parque, para definir e dar suporte a ações concretas que fortaleçam a cooperação e a colaboração entre essas instituições nos temas de interseção.

Outra estratégia no planejamento do PTI tem sido a atuação de sua Fundação responsável, com a inclusão da missão de buscar a promoção e o fomento do desenvolvimento científico, tecnológico e da inovação no Parque, configurando um sistema de CTI nesse ambiente. Para tanto, vem desenvolvendo e consolidando as chamadas Plataformas de Ciência, Tecnologia e Inovação (Marques e Sotuyo, 2014) que se constituem ambientes de uso compartilhado de infraestruturas e recursos para as diversas instituições instaladas no Parque e no território. O Parque viabiliza espaços de interação não apenas da ciência e tecnologia, mas também de participação dos indivíduos e entidades, podendo ser um elemento promotor da integração política institucional, educacional e sociocultural do território onde está instalado. O objetivo é induzir e promover um processo de desenvolvimento cooperado e colaborativo de ciência, tecnologia e inovação para a geração do desenvolvimento socioeconômico.

Em 2015, a FPTI promoveu o primeiro Colóquio da Política de CTI do Parque. Essa iniciativa, ainda em curso, tem como objetivo a elaboração participativa da Política de CTI do PTI, de modo a congregar todos os atores, capturar as diversas percepções e buscar atender as demandas e anseios e o bem comum, por meio de uma política de CTI. Essa ação é parte do Planejamento Estratégico da FPTI e se estrutura a partir de diversas interações de grupos de atores que ao longo do tempo vão definindo pontos de interesse e coelaborando a política de CTI para o Parque. Os atores foram organizados conforme temáticas da Tabela 3.

\section{Quadro 1 - Temáticas desenvolvidas pelos grupos do PTI}

\begin{tabular}{|llll|}
\hline Educação & Pesquisa & $\begin{array}{l}\text { Institucionalidade } \\
\text { das relações }\end{array}$ & Tecnologia e Desenvolvimento \\
Desenvolvimento & Problemáticas & Acordo de PI & Prototipagem \\
Territorial & Grau de relevância & Aspectos de comunicação & Unidades demonstrativas \\
Cultura & Condições para pesquisa & Disseminação & Sistemas piloto \\
Extensão & Criação de um ambiente & Questões legais & Ensaios e testes comprobatórios \\
Formação de RH & científico e tecnológico & Estudo de mercado \\
\hline
\end{tabular}

Fonte: Elaborada pelos autores

Os principais pontos destacados pelos atores durante o evento estão compilados em três grupos distintos: a) principais percepções dos atores em relação ao seu papel no sistema de CTI do Parque, b) principais percepções dos atores em relação ao papel do PTI e de sua entidade gestora - FPTI em relação ao seu papel no sistema de CTI do Parque, e percepções sobre pontos comuns entre todos os atores, conforme Tabela 4.

\section{Quadro 2 - Percepção dos atores em relação aos papéis no Sistema de CTI do Parque}

\section{Em relação ao seu papel}

Formação de recursos humanos/profissionais qualificados para atuarem na região

Complementariedade dos cursos existentes nas universidades.

Projetos podem promover o desenvolvimento de qualidade da região, aproveitando os potenciais da Instituições envolvidas.

Pesquisa básica e tecnológica na área de materiais, energias e biodiversidade.

\section{PRINCIPAIS PERCEPÇÕES}

Em relação ao papel do PTI

Criação de ambiente científico e tecnológico.

Valorização efetiva do trabalho do estudante e demais profissionais.

Fomento à pós-graduação; pesquisa básica e tecnológica na área de materiais, energias e biodiversidade. Tópico energia com um dos eixos estruturantes e estratégicos de pesquisa no PTI.

Fortalecer os grupos de pesquisa, observando uma governança para atender a interinstitucionalidade própria do PTI. 
Desenvolvimento de projetos com a comunidade acadêmica de Ensino Básico na região, na sua formação de científica, projetos culturais na região como cinema, música e teatro.

Desmitificar a área de desenvolvimento como sendo de "segunda-classe", embora as Universidades sejam cobradas para a elaboração.

Estreitar os laços de pesquisa e desenvolvimento entre as instituições e a partir disso, buscar as ações conjuntas que devem ser tomadas para melhorar o sistema de pesquisa, desenvolvimento e ensino como um todo.

Buscar um interlocutor em cada instituição.
Fortalecer e fomentar a aplicação de ferramentas de simulação numérica computacional dentro do PTI.

Ter espaços e laboratórios dedicados à prototipagem de produtos desenvolvidos no PTI.

Desenvolver estratégias para alinhar os interesses das instituições que "habitam" o PTI.

Criar mecanismos ou estratégias de comunicação para interação efetiva entre atores PTI.

\section{PONTOS DE TRABALHO COMUM}

Fortalecer o trabalho interinstitucional começando pela identificação de áreas.

Buscar a criação de políticas comuns dentro das instituições para a realização de pesquisa.

Integrar de forma efetiva uma política: projetos, universidades, estudantes, iniciação científica e tecnológica, fomento

Abertura dos projetos, através de workshops dos projetos da FPTI e Universidades.

Mapear as competências / desenvolvimento.

Fazer a gestão integrada do conhecimento gerado no PTI.

Definir estratégias, processos e procedimentos para chegar ao mercado.

Necessidade de mudança cultura.

Fonte: elaborado pelos autores, 2016.

Em relação ao Framework ReCIS, buscou-se, neste trabalho, verificar o seu potencial de aplicabilidade em um ambiente do PTI. Em relação às dimensões de análise, o fator Desenvolvimento Regional pode ser considerado, também, fator de Geração de Valor, dado que um Parque Tecnológico é um mecanismo promotor do desenvolvimento regional, que por meio de suas atividades e espaços integra e estimula a CTI.

Além dessa mudança de perspectiva sobre a dimensão de desenvolvimento regional, verificamos como recomendável a adequação e mudança dos seguintes critérios:

- Institucionalização: Adicionar os critérios: Transparência, Participação: grau de colaboração, cooperação e coprodução e Relações interinstitucionais, tendo em vista que um dos principais papéis do Parque é promover a integração, colaboração e coprodução dos diversos atores nas diferentes instituições, seja intra ou extraparque. A transparência e as relações interinstitucionais são fatores fundamentais para o estabelecimento de relações de confiança e troca de informações e conhecimento.

- Infraestrutura: Adicionar os critérios: Infraestrutura laboratorial e de suporte, TICs, Política de CTI e Fomento para PD\&I. Muito embora os atores do Parque tenham a sua disposição um conjunto de laboratórios e serviços de suporte e TICs de qualidade, as demandas são crescentes em infraestruturas específicas, a exemplo de laboratórios de prototipagem. Também no caso do Parque, a existência de uma política de CTI e fomento à PD\&l são fatores essenciais.

- Mercado: Adicionar os critérios: Mecanismos de criação e desenvolvimento de empresas, Espaços de Coworking e Coprodução, Fomento à PD\&l, Sistema de captação de recursos, Antena Tecnológica/Observatório. Como um ambiente promotor da inovação, os aspectos relacionados a mercado são os que mais necessitariam de adequação. Nesse caso, é importante avaliar a disponibilidade de espaços e mecanismos de criação e desenvolvimento de empresas, a disponibilidade e acesso a informações estratégicas de mercado, tecnologias e produtos, e a disponibilidade e acesso a fomento para PD\&l.

- Educação: Um dos aspectos mais destacados na percepção dos atores é o papel das instituições no processo de formação, seja pela educação formal ou não formal. Nesse sentido, sugere-se também a avaliação do grau de integração do Parque com a educação básica, além da disponibilidade de cursos de graduação e pós-graduação. Quando se considera a inserção de um Parque Tecnológico na sociedade, o quanto que este ambiente contribui na disseminação das ciências. 
Tabela 3 - Dimensões, fatores e critérios do Framework aplicados a um Parque Tecnológico

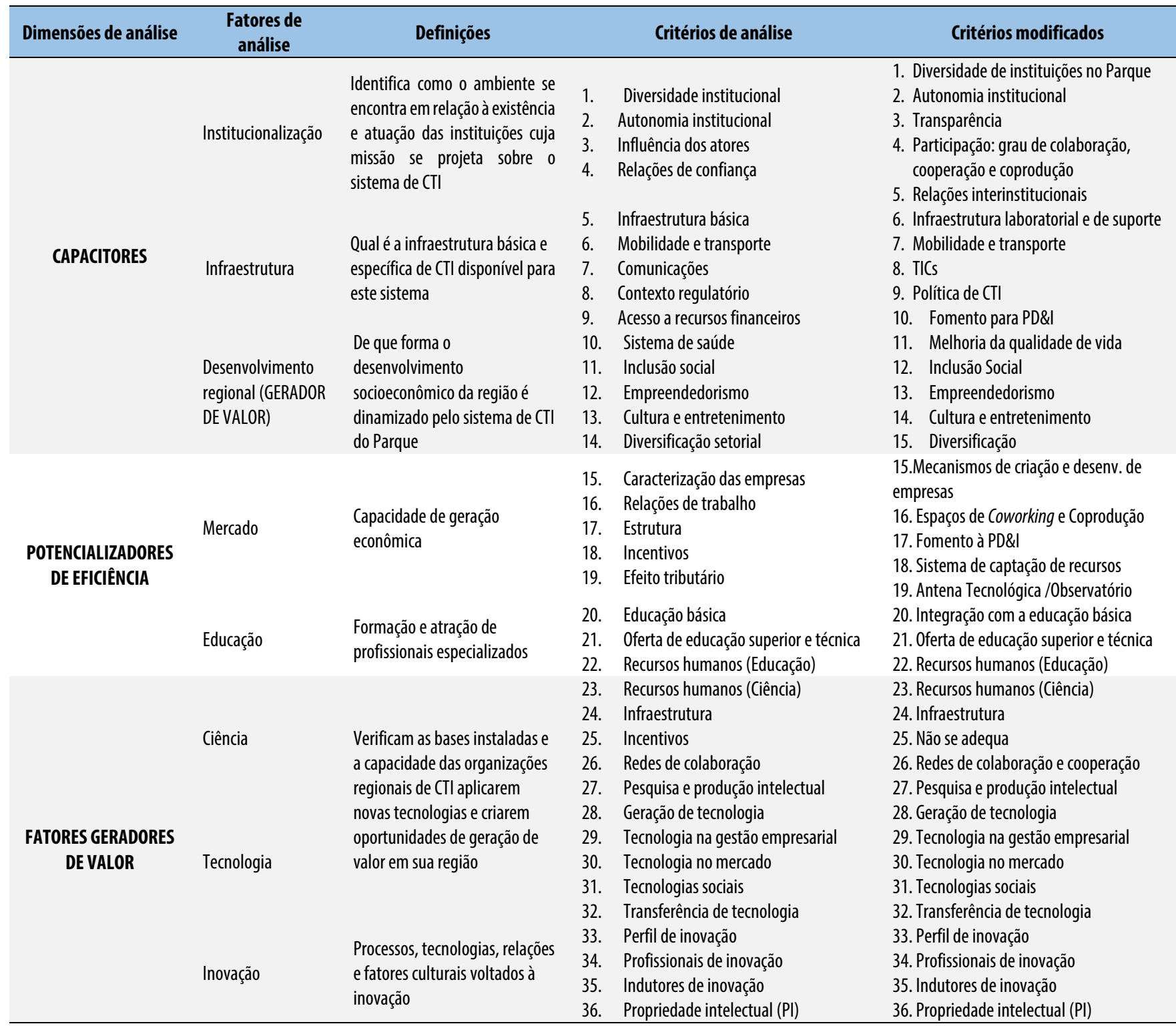

Fonte: elaborado pelos autores, 2016.

\section{CONSIDERAÇÕES FINAIS}

O estudo permitiu identificar a pertinência das dimensões de análise a serem aplicadas tanto no contexto de outro estado da federação, bem como, em um habitat de inovação como um Parque Tecnológico. As Dimensões do Framework se configuram como uma base sólida para relacionar os critérios adotados. Tendo o foco de análise em dois ambientes diferentes, porém, que agregam a sinergia de questões relacionadas com CTI, os critérios de análise tenderam a sofrer modificações para equacionar as realidades locais.

Percebe-se que para aplicação em outro estado, seu contexto está mais alinhado que no contexto de um parque tecnológico, onde não apenas os critérios de análise foram modificados, mas também os fatores de análise. Especificamente em um ambiente de Parque, as análises relacionadas aos fatores Institucionalização, Infraestrutura e Mercado poderiam sofrer maiores alterações devido ao papel que um ambiente dessa natureza desempenha em um determinado território. Nesse sentido, as percepções dos atores estão muito relacionadas à dinâmica de interação e interrelacionamento das instituições, ao grau de confiança e de conhecimento da diversidade institucional, além das necessidades de recursos físicos e financeiros, o que abre oportunidade para estudos mais aprofundados a respeito.

Para fins de aprofundamento na análise de aplicação em diferentes unidades identifica-se a necessidade de abordar outros aspectos do Framework como, por exemplo, o conjunto de questões elaboradas para os atores, além dos critérios de análise, foco deste estudo. 
Desta forma, considera-se que o Framework teria a possibilidade de ser adotado e aplicado para elencar e avaliar as percepções em outras unidades de análise, sendo recomendado estudos mais aprofundados. Ainda que este estudo tenha sido limitado, entende-se que o Framework poderia representar uma ferramenta de grande valor para auxiliar governantes e gestores públicos e privados na formulação de políticas de CTI em diversos contextos.

\section{BIBLIOGRAFIA}

Alves, V. C. (2013). Impactos da gestão do conhecimento nos núcleos de inovação de tecnológica- NIT das Universidades Estaduais da Bahia. Dissertação de Mestrado, Senai Cimatec, Salvador.

BAHIA. (s.d.). SECTI. Acesso em 03 de Agosto de 2016, disponível em http://www.secti.ba.gov.br/arquivos/File/DECRETOS_E_LEIS/LEI8897.pdf

IBGE. (s.d.). Acesso em 15 de Julho de 2016, disponível em Instituto Brasileiro de Pesquisa e Estatística: http://www.ibge.gov.br/home/presidencia/noticias/pdf/analise_estimativas_2014.pdf

Kable, J. C. (Março de 1979). Decision Perception Analysis. Journal of Human Resources, 16, pp. 39-47.

Kable, J. C. (1983). Decision Perception Analysis-Measuring A Manager's Preference for Managing. Journal of management development, 2, pp. 3-18.

Lastres, H. M., Lemos, C., Falcón, M. L., Andrade, J. E., Magalhães, W., \& Silva, M. M. (2014). O apoio ao desenvolvimento regional: a experiencia do BNDES e oportunidades para avanços. Revista do BNDES, 5-46.

Marques, M. A., \& Sotuyo, J. C. (2014). Plataformas de C,T\&l para geração de inovação na promoção do desenvolvimento territorial - O caso do Parque Tecnológico Itaipu. IV Congreso Internacional de Gestión Tecnológica e Innovación, (p. 15). Cartagena, Colombia.

MCTI. (2016). Estratégia Nacional de Ciencia, Tecnologia e Inovação 2016-2019. MInistério da Ciência Tecnologia e Inovação, Brasilia.

Pacheco, R. C., Selig, P. M., \& Zucco, C. (2016). Conferência Estadual de Ciência, Tecnologia e Inovação de Santa Catarina: metodologia e resultados para o plano estadual de CTI. Florianópolis: Instituto Stela.

Santa Catariana. (15 de Janeiro de 2008). FAPESC. Fonte: http://www.fapesc.sc.gov.br/wpcontent/uploads/2015/09/03092009lei_inovacao.pdf

Santa Catarina. (s.d.). Acesso em 15 de Julho de 2016, disponível em Conferencia Estadual de CTI: http://www.conferenciacti.sc.gov.br/?page_id=13319

Santa Catarina. (s.d.). Acesso em 19 de Julho de 2016, disponível em Secretaria de Estado de Desenvolvimento Sustentável: www.sds.sc.gov.br/cecop/index.php/download/doc.../22-lei-n-14-328-2008

SECTI - Secretaria de Ciencia Tecnologia e Inovação do Estado da Bahia. (2010). Acesso em 28 de julho de 2016, disponível em Portal do livro aberto em CTI: http://livroaberto.ibict.br/bitstream/1/724/13/Estadual\%20Bahia.pdf

SECTI. (2004). Politica de Ciencia Tecnologia e Inovação para o Estado da Bahia. Salvador. 